International Journal of Engineering \& Technology, $7(3.1)(2018)$ 171-173
International Journal of Engineering \& Technology
SPC
Website: www.sciencepubco.com/index.php/IJET
Research paper

\title{
Remote Access of Medical Image Processing and 3D Visualization Application using Raspberry Pi
}

\author{
Sreepathy $\mathrm{H} \mathrm{V}^{1}$, Nandish $\mathrm{S}^{2^{*}}$, Chaitanya $\mathrm{CVS}^{3}$ \\ ${ }^{1}$ School of Information Sciences, Manipal Academy of Higher Education, India \\ *Corresponding author E-mail: nandish.s@manipal.edu
}

\begin{abstract}
With the extensive growth in technology, healthcare sector has benefitted a lot recently. Looking into the academic research and validation in the area of medical image processing and visualization, many platforms and the open-source resources are available. Insight toolkit (ITK) and visualization toolkit (VTK) are extensively used for medical image processing and 3D visualization respectively. Resources used to develop an application using ITK-VTK and same resources be used to deliver it to the users such as, clinicians, doctors etc. This can be achieved by using respective hardware and the infrastructure. In the proposed article, the infrastructure and resource used to build and deploy the application and remote access given to the users are elucidated.
\end{abstract}

Keywords: Java Client; Raspberry Pi; VTK; X server

\section{Introduction}

This Medical image processing and 3D visualization have extensive applications for better diagnosis, treatment and care. Most of the researchers in academic set up is working in similar area. These application requires a better infrastructure and resources for development. Insight toolkit (ITK) and Visualization toolkit (VTK) are the open source packages dedicated for medical image processing and 3D visualization respectively. ITK-VTK supports different platforms and hardware for developing rapid prototypes. Most of the academic institutions may not have efficient infrastructure to develop a $3 \mathrm{D}$ visualization application with volume rendering and depth peeling. It requires better systems with graphics support. Such applications ae also very much need in small radiological clinics. Proposed paper elucidates the work done to achieve development and deployment of medical imaging application with low resource setting with sufficient graphic support. ITK is used for processing the medical images and VTK is used to perform $3 \mathrm{D}$ volume rendering of medical images before and after processing respectively. Instead of building VTK in the $\mathrm{PC}$, in processed literature VTK is built on RaspberryPi3 with Ubuntu. Required example for volume rendering is also built on the same system and given access to users for validating the resultant of the application. Medical data used in proposed system are in DICOM format. Computed Tomography (CT), Magnetic Resonance Imaging (MRI), Ultrasound (US) and X-rays are commonly heard medical images. CT brain scanned images are used in proposed work. Figure 1 and 2 shows CT and MRI brain images respectively in axial, coronal and sagittal planes. Number slices may vary from 25 to 1000 approximately depending the cases. So the system trying to build in proposed article should also take care of the input load from the data. CT and MRI images of any part of the images can be used in the proposal. CT images gives better characterization of bones and vacuum present in the human body and MRI gives better characterization of soft tissues and fluids in human body.

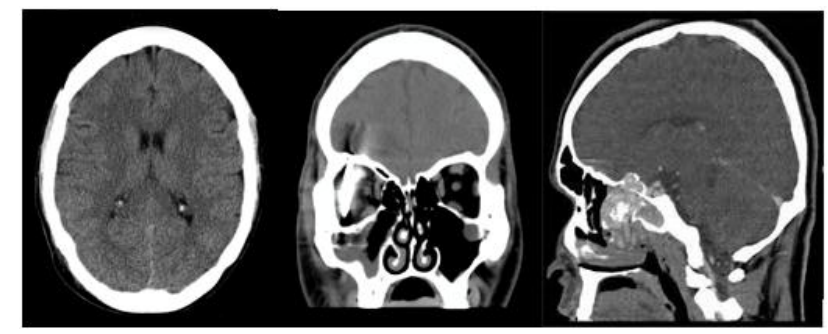

Figure 1: CT Brain axial section (Left) Coronal (Middle) Sagittal (Right)

VTK is used for volume rendering of medical images. VTK has its pipeline for visualization and same is used. The application developed in a PC, if required to deliver it for the users for validation and testing, same infrastructure and required software with graphics support is needed in user PC. User's workstation and the PCs are not preferred to use for validating and testing the prototypes developed.

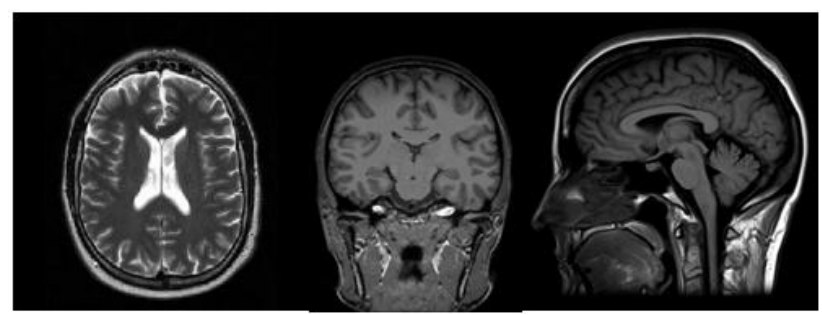

Figure 2: MRI brain axial section (Left), coronal (Middle) and Sagittal (Right).

To address these issues and have a smooth conduction of validation and testing of prototypes, remote access to the development environment is required. Since rendering of the medical data and available remote desktop access may not provide required infrastructure to deliver the work for the users. Hence it is recommended and tried to develop an application on RaspberryPi3 and pro- 
vide remote access within local area network may solve replicating the software and environment set up at user's PC.

\section{Methodology}

Proposed methodology is as shown in figure 1 and 2 respectively for visualization pipeline used in VTK and hardwareinfrastructure proposed respectively.

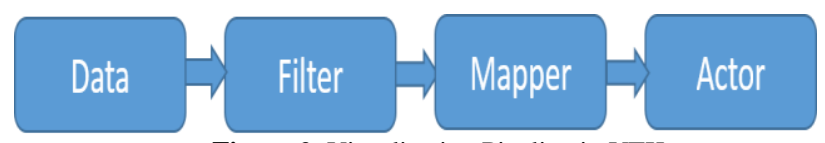

Figure 3: Visualization Pipeline in VTK

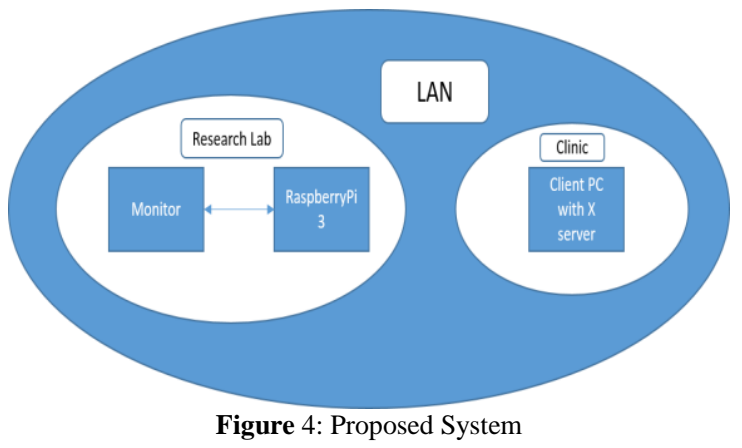

Explanation for pipeline for visualization used in VTK can be referred from [1,2]]. Proposed system RaspberryPi3 with the GPU 'Broadcom VideoCore IV', four high-performance ARM CortexA53 processing cores and 1GB RAM is used along with HDMI monitor. Raspbian Stretch OS is ported on the RaspberryPi3 and VTK-7.1.1 is configured and built on the same. Entire system is built within the LAN; development of the application is performed in lab dedicated for the research activities provided in Manipal Academy of Higher Education. Proposed system, Linux is used initially for the remote access of RaspberryPi3 suing command below,SSH -X user/host_name. It is successfully rendered the 3D volume remotely by enabling $\mathrm{X}$ server. For windows Java client is installed to enable the SSH. In Linux OS the drivers are updated automatically, hence mesa and openGL was updated in Linux supporting 3D rendering and Depth peeling. Some remote desktop solutions such as VirtualGL for UNIX allow remote visualization with full OpenGL support [3]. But most of the users may not be familiar with UNIX and Linux OS. In windows 10 OS, X server enabling is the issue. To resolve this issue and third party $\mathrm{X}$ server is installed and it should be enabled through the Java client used in proposed system. Hence the $3 \mathrm{D}$ volume rendering of input data and processed medical images are performed from the client machine. Remote volume rendering allows the sharing of expensive remote graphics hardware via the Internet by using a simple client computer. By connecting more clients to the rendering server, the collaboration of many experts from different locations is possible [4].

\section{Results and Discussion}

Above mentioned methodology for visualization pipeline in VTK uses GPURenderDemo.cpp example available in VTK 7.1.1 package. Recent version of VTK 8 is also released.

Figure 6 shows volume rendering of CT input data of the brain. CT data of head and neck used, consists 361 slices, each with 1.5 $\mathrm{mm}$ thickness and 0.7 spacing between slices and voxel size of $0.42 \times 0.42 \times 0.70$. Data is taken from osirix image database [1].

Figure 6 shows 3D volume rendering and depth peeling CT head and neck data taken from osirix image database with the voxel size $0.71 \times 0.71 \times 1.50 \mathrm{~mm}$ and image width and height of $364 \mathrm{~mm}$ respectively. Data consists of thoracic and abdomen regions. Preprocessing of data follows selection of only thoracic region (199 slices of thoracic region selected manually) and resizing the image from $512 \times 512$ to $256 \times 256$ [5].

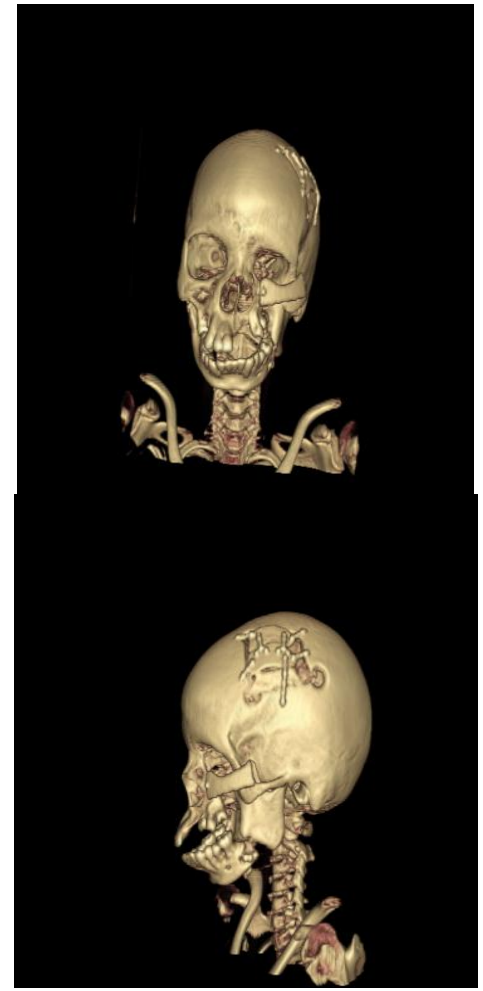

Figure 5: Bone volume rendering of brain $\mathrm{CT}$.

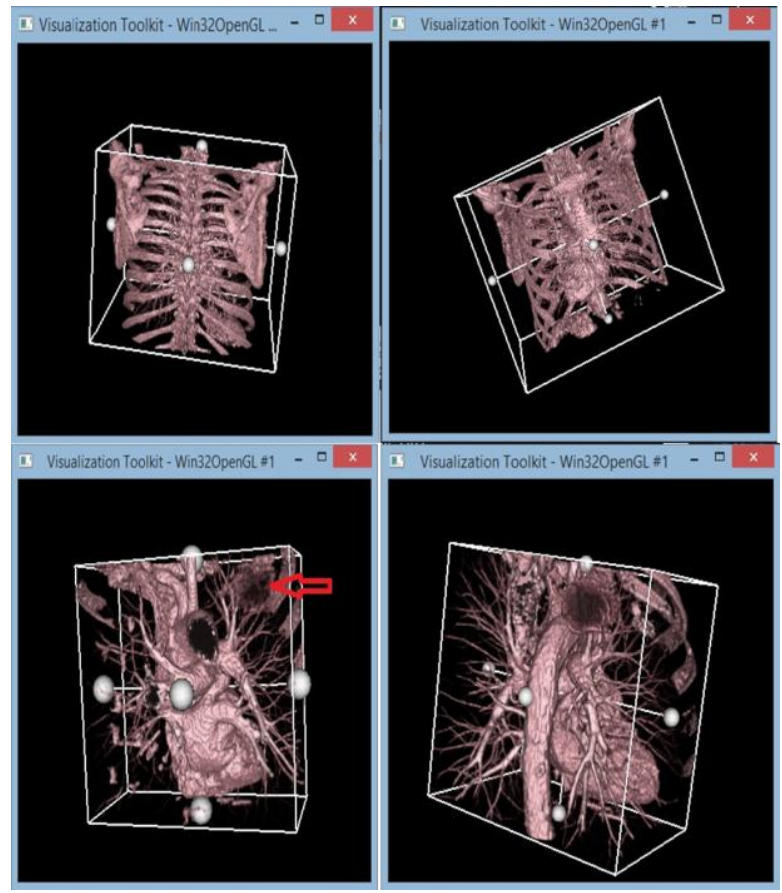

Figure 6: 3D Volume rendering and Depth Peeling of processed CT lung images and pointing carcinoma in bottom left image.

Above results shows volume rendering of CT data of brain and lung region using VTK. Entire process of configuring and building VTK and the examples requires minimum $3 \mathrm{~GB}$ of space in Windows PC. Prototype of the application developed should be validated and tested by the user. Repeating the same process of configuring and building VTK and project in the user's PC is again having to consume same amount of space and infrastructure. So the proposed system may solve the issue and can be connected through LAN and user can be access is remotely. If the user is from other network RaspberryPi3 where application is developed can be connected to the respective LAN and same can be accessed without any extra requirement of the infrastructure. Figure 7 
shows RaspberryPi3 setup and volume rendering of CT brain data at development side.

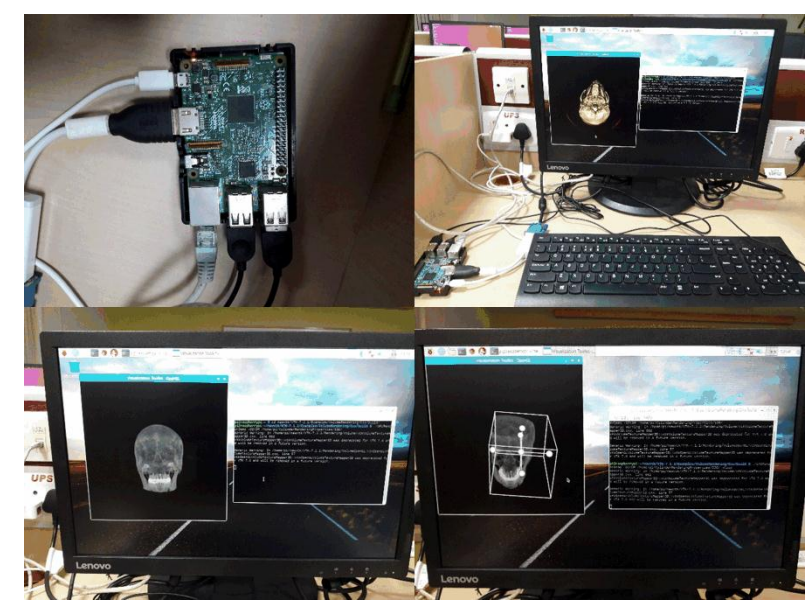

Figure 7: RaspberryPi3 Board (Left Top), Raspberry setup and Display (Right Top), 3D rendering and Depth Peeling (Bottom)

Figure 8 shows accessing raspberry remotely from client side enabling X server using java client.

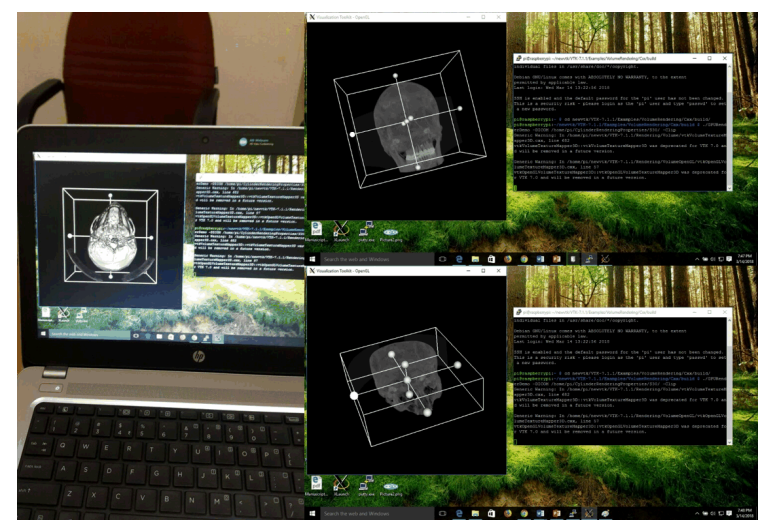

Figure 8: Client System accessing remotely (left), 3D volume rendering and Depth peeling (Right top and bottom respectively)

\section{Conclusion}

Applications development and research in the area of medical image processing and 3D visualization is trendy largely across academic institutions and organizations. Proof of concept should be validated and tested at the user site. Users for such applications are doctors, clinicians and other practitioners in healthcare. Most of academic research takes place using open-source packages, dedicated software and low resource setting unlike in industries. Proposed system with remote access of medical image application on 3D visualization requires good graphics support in the development environment. User workstations may not be identical to deploy the applications developed on different environment. Thus proposed system using RaspberryPi3 with GPU and CPU with 1GB RAM having Raspbian Stretch OS provides platforms to develop applications on 3D visualization of medical images such as CT, MRI. And also gives remote access from client machine by enabling X server through Java client. Future work for the proposed system would be to build a software package with GUI on the same environment and providing access to the users remotely. And providing more clinical data for validation is required, users can send images to the existing system using DICOM cleaner. DICOM cleaner is an open source tool with GUI which is used to import, clean (Anonymize) and send to remote device through network (LAN).

\section{References}

[1] Nandish S, Gopalakrishna Prabhu, K.V. Rajagopal, "3D Visualization of Brain CT and MRI Sequence Using VTK", JARDCS, Special Issue 13, pp. 854-858, December 2017

[2] The VTK User's Guide, 11th Edition, Kitware, Inc, 2010.

[3] Roland Unterhinninghofen, Frederik Giesel, R“udiger Dillmann, "MEDIFRAME - Remote Volume Rendering Visualization Framework", Proceedings of IEEE EMBS, 33rd Annual International Conference, pp. 2368-2371, September 2011.

[4] Bernd F. Tomandl, MD,Peter Hastreiter, Christof RezkSalama, ,Klaus Engel, et.al, "Local and Remote Visualization Techniques for Interactive Direct Volume Rendering in Neuroradiology", Radiographics, Volume 21, No. 6, pp. 15761-1572, December 2001.

[5] Nandish S, Gopalakrishna Prabhu K, Rajagopal KV, Mohandas Rao K G, Manjunath Shetty, "3D Visualization and Dept Peeling of Pulmonary Vasculature and Lung Lesion Using Plain CT Sequence", IEEE Fourth International Conference on Image Information Processing, pp. 34-37, December 2017 